5 - ORIGINAL ARTICLE

ISCHEMIA-REPERFUSION

\title{
Allopurinol preconditioning attenuates renal ischemia/reperfusion injury by inhibiting HMGB1 expression in a rat model ${ }^{1}$
}

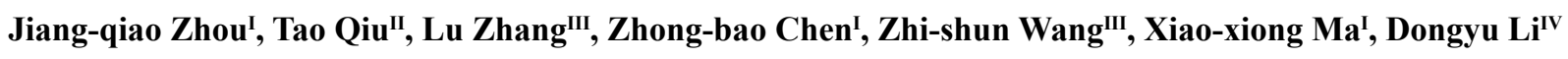 \\ DOI: http://dx.doi.org/10.1590/S0102-865020160030000005 \\ IPhD, Department of Organ Transplantation, Renmin Hospital of Wuhan University, Wuhan, Hubei, China. Conception and design of the study, \\ acquisition and analysis of data, manuscript writing. \\ IIPhD, Department of Organ Transplantation, Renmin Hospital of Wuhan University, Wuhan, Hubei, China. Design and supervised all phases of the \\ study, critical revision. \\ IIIPhD, Physician, Department of Urology, Renmin Hospital of Wuhan University, Wuhan, Hubei, China. Acquisition of data. \\ ${ }^{\text {IV }}$ Physician, Intensive Care Unit, PuAi Hospital, Anlu, Hubei, China. Helped processing figures.
}

\begin{abstract}
PURPOSE: To investigate the potential effects of pretreatment with allopurinol on renal ischemia/reperfusion injury (IRI) in a rat model.

METHODS: Twenty four rats were subjected to right kidney uninephrectomy were randomly distributed into the following three groups ( $\mathrm{n}=8$ ): Group A (sham-operated group); Group B (ischemic group) with 30 min of renal ischemia after surgery; and Group C (allopurinol + ischemia group) pretreated with allopurinol at $50 \mathrm{mg} / \mathrm{kg}$ for 14 days. At $72 \mathrm{~h}$ after renal reperfusion, the kidney was harvested to assess inflammation and apoptosis.

RESULTS: Pretreatment with allopurinol significantly improved renal functional and histological grade scores following I/R injury $(p<0.05)$. Compared with Group B, the expression levels of caspase-3 and Bax were markedly reduced in Group C, meanwhile, whereas expression of bcl-2 was clearly increased $(\mathrm{p}<0.05)$. A newly described marker of inflammation, High Mobility Group Box $1(\mathrm{HMGB} 1)$, showed reduced expression in Group $\mathrm{C}(\mathrm{p}<0.05)$.
\end{abstract}

CONCLUSION: Pretreatment with allopurinol had a protective effect on kidney ischemia/reperfusion injury, which might be related to the inhibition of HMGB1 expression.

Key words: Inflammation. Ischemia. Reperfusion. Allopurinol. Apoptosis. Rats. 


\section{Introduction}

Renal IRI contributes to the quality of a kidney graft, which has a direct association with the survival of the recipient. A major factor for the success of a kidney graft is the warm ischemic time (WIT). Uric acid is an end-product of adenosine triphosphate (ATP) metabolism. During a long period of ischemia, a series of oxidation-reduction reactions occur whereby ATP is successively degraded to adenosine, inosine, xanthine, and hypoxanthine, while xanthine dehydrogenase is converted to xanthine oxidase (XO) when exposed to ischemia-induced cellular calcium overload ${ }^{1}$. In the reoxygenation of ischemic tissues, many types of reactive oxygen species (ROS) accumulate in renal tubular and vascular endothelial cells. ROS can 'attack' cells and affect renal function. Several previous studies have reported that some inhibitors of XOX (e.g., allopurinol) and other antioxidant agents can have a protective effect on injury induced by renal ischemia reperfusion ${ }^{2-4}$. Some other studies have proposed that ischemic reperfusion injury is not caused by XOX-generated ROS reoxygenation.

Recent studies have suggested that the initial damage to cells along with the activation of inflammatory signaling are involved the release of endogenous damage-associated molecular pattern (DAMP) signaling molecules from damaged/ischemic cells or IRI tissues. High mobility group box 1 (HMGB 1), a known DAMP molecule, can trigger cell-mediated inflammatory responses by enhancing the expression of TLR4 and increasing reactivity to it and other DAMP molecules ${ }^{5}$. HMGB1 was considered to be a novel key molecule during the IRI after $\mathrm{KTx}^{6}$. After it is released, HMGB1 protein can bind to its receptors that are expressed on effector cells to induce inflammation, cell proliferation, and chemotaxis, thereby acting as a bridge between the innate and adaptive immune responses ${ }^{7}$. HMGB1 is a multi-functional regulatory molecule that can be actively secreted in response to endogenous inflammatory stimuli (e.g., TNF- $\alpha$ ). Extracellular HMGB1 can activate NF- $\kappa B$ and induce the translocation of other nuclear translocation, which results in increased release of additional pro-inflammatory cytokines, such as TNF- $\alpha$, IL-6, and HMGB1 itself via a positive feedback loop that involves NF- $\kappa \mathrm{B}$ acting on its own target receptors ${ }^{8}$. The signaling pathways upstream and downstream of HMGB1 remain unknown. Considering the multiple roles of HMGB1 in various pathologies, inhibition of HMGB1-mediated signaling may represent a potential effective therapeutic approach ${ }^{7}$. The study of Rabadi and colleagues showed that UA, which is considered to be an additional early response-signaling molecule both in vitro and in vivo, could induce the secretion of HMGB1 by endothelial cells ${ }^{9}$.
Allopurinol is a XOX inhibitor and antioxidant free radical scavenger, which is administered as a medication that is primarily used to treat excess uric acid (UA) in the blood and reduce its associated complications, including chronic gout. High-dose allopurinol can improve endothelial function by profoundly reducing vascular oxidative stress ${ }^{10}$. Its adaptive metabolite, oxypurinol, can provide some protection against $\mathrm{I} / \mathrm{R}$ injury by suppressing the production of ROS and $\mathrm{Ca}^{2+}$ overload ${ }^{11}$. Allopurinol has been reported to attenuate reperfusion injury in various organs ${ }^{12}$. Moreover, tubular cell apoptosis has been found to be a major factor involved in the mechanism of renal IR injury ${ }^{13}$. Therefore, therapeutic approaches aimed at suppressing inflammatory responses and tubular apoptosis have been recognized to represent effective measures that may act to prevent renal injury and offer better prognosis after an I/R insult ${ }^{14}$.

UA released after IRI can mediate the acetylation and release of HMGB1 from endothelial cells by several mechanisms, so that administration of allopurinol may represent another way to relieve inflammation by inhibiting UA release and HMGB1 nuclear translocation. This study aimed to test the hypothesis that pretreatment with allopurinol may provide protective effects on renal IRI by inhibiting HMGB1 expression.

\section{Methods}

The animal experiments were performed according to the guidelines of the Chinese Council for Animal Care.

Male, adult Sprague Dawley (SD) rats (body weight, 250-300 g) were obtained from the Experimental Animal Center of the Medical College of Wuhan University (Wuhan, China). Animals were maintained at the Central Animal Facility of the Affiliated Renmin Hospital of Wuhan University. Rats were maintained under air-filtered, constant temperature $\left(20-22^{\circ} \mathrm{C}\right)$ conditions in a light-controlled room (light for 7 a.m. to 7 p.m.), and were allowed free access to a standard diet.

\section{Experimental protocol}

Briefly, rats were anesthetized by intraperitoneal injection with pentobarbital $(50 \mathrm{mg} / \mathrm{kg})$. Rats were placed on an electric heating pad to maintain a body temperature of $37^{\circ} \mathrm{C}$. A right nephrectomy was performed. The left renal artery and vein were blocked using a vascular clamp for $30 \mathrm{~min}$ to induce ischemia and the left kidney was reperfused for $72 \mathrm{~h}$. Finally, all rats were killed.

Rats were separated into the following three groups. In Group 1, sham operated control rats $(n=8)$ were subjected to right nephrectomy, but without the induction of left renal ischemia. In 
Group 2, ischemia/reperfusion (I/R) rats $(n=8)$ were subjected to right nephrectomy and left renal ischemia for $30 \mathrm{~min}$, followed by a $72 \mathrm{~h}$ reperfusion period. In Group 3, rats treated with allopurinol preconditioning $+\mathrm{I} / \mathrm{R}(\mathrm{n}=8)$, prior to $\mathrm{I} / \mathrm{R}$ manipulation (as in group 2), were administered allopurinol $50 \mathrm{mg} \bullet \mathrm{kg}^{-1} \bullet \mathrm{day}^{-1}$ (Sigma, St. Louis, MO, USA) $\mathrm{IP}^{15}$ for two weeks, as described previously ${ }^{2}$, and then were subjected to the same manipulations as group 2 .

\section{Serum assays}

Rats were killed $72 \mathrm{~h}$ after reperfusion. Blood samples were obtained from the inferior vena cava to measure the concentration of urea nitrogen (BUN) and creatinine (Cr) before surgery. BUN and $\mathrm{Cr}$ in blood samples were analyzed using standard techniques with an Olympus AU 2700 Analyzer (Olympus Optical Co., Tokyo, Japan). Levels of MPO and SOD were detected using a standard procedure with a colorimetric activity assay kit (Beyotime, Nanjing, China).

\section{Histological examination}

The left kidney was excised $72 \mathrm{~h}$ after reperfusion. After excision, the kidney was fixed with $10 \%$ phosphate buffered formalin, paraffin embedded, and sectioned to a 4-mm thickness according to a standard procedure ${ }^{16}$. Sections were deparaffinized and gradually hydrated before they were examined by H\&E staining. Morphological assessments were performed by an experienced renal pathologist who had not been informed of the experimental protocol. The Jablonski scoring system was applied for histopathologic assessments of $\mathrm{I} / \mathrm{R}$ induced damage of the proximal tubules ${ }^{17}$.

\section{Immunohistochemistry}

Sections were prepared according to the manufacturer's guidelines for immunohistochemical assays (Gene Tech, Wuhan, China). Levels of HMGB1 protein expression in each group were analyzed and evaluated based on the staining intensity (negative, mild, or strong) by microscopy.

\section{Western blotting}

Proteins were extracted from kidney tissues according to standard protocols. In brief, protein samples were separated on $12.5 \%$ sodium dodecyl sulfate-polyacrylamide gel electrophoresis gels $(40 \mu \mathrm{g} / \mathrm{lane})$, and then were transferred to a nitrocellulose membrane (Bio-Rad). The membrane was blocked with 5\% non-fat dry milk in TBS-T buffer, and then incubated with rabbit polyclonal anti-Bax (1:1000; Cell Signaling Technologies [CST], Danvers, MA, USA), Bcl-2 (1:1000; CST, CA), Caspase3 (1:1000; CST, CA), TGF- $\beta 1$ (1:1000; CST, CA), HMGB1 (1:800; CST, CA), or Smad3 (1:2000; abcam, Cambridge, UK) antibody or mouse monoclonal anti-GAPDH antibody (1:1000; Santa Cruz, Dallas, TX, USA) overnight at $4^{\circ} \mathrm{C}$. After extensive rinsing with TBS-T buffer, blots were incubated with HRP-conjugated anti-rabbit or anti-mouse secondary antibodies (Santa Cruz) and developed using an enhanced chemiluminescence system (ECL Kit; Pierce Biotechnology Inc., Rockford, IL, USA) and captured on light-sensitive imaging film (Kodak, Tokyo, Japan).

\section{TUNEL staining}

To detect apoptosis induced by ischemia, an in situ apoptosis detection kit (Promega, Madison, WI, USA) was used. The TUNEL assay was performed according to the manufacturer's instructions with several modifications. In brief, whole tissue samples were fixed in $4 \%$ paraformaldehyde/PBS (pH 7.4) solution at $4^{\circ} \mathrm{C}$ overnight. After three washes with $1 \times$ PBS solution, samples were immersed in $70 \%$ ethanol for at least $24 \mathrm{~h}$ at $20^{\circ} \mathrm{C}$. After the samples were again washed three times with $1 \times$ PBS, they were incubated with permeabilization buffer for $15 \mathrm{~min}$ on ice and followed by a PBS wash. Then, samples were transferred into $50 \mathrm{~mL}$ Reaction Buffer (TdT Enzyme $5 \mathrm{~mL}+$ Labeling Safe Buffer $45 \mathrm{~mL}$ ) for a 90 -min incubation at $37^{\circ} \mathrm{C}$. The labeling procedure was stopped by washing with PBS solution. Staining results were analyzed based on the percentage of cells with positive TUNEL staining that were called using American Image-Pro Plus software.

\section{Statistical analysis}

Statistical analyses were performed using SPSS version 17.0 (SPSS Inc, Chicago, IL, USA). One-way ANOVA with a posthoc test (Bonferroni test, which compared all pairs of columns) was used to analyze all data. A $p<0.05$ was used as a threshold for statistically significant differences.

\section{Results}

\section{Allopurinol preconditioning reduced renal dysfunc- tion after IRI}

Compared with the $\mathrm{I} / \mathrm{R}$ group, the allopurinol preconditioning group had significantly reduced levels of MPO and increased levels of SOD. Moreover, allopurinol significantly 
reduced levels of BUN and serum creatine following IRI. might reduce oxidative damage and improve renal function after Together, these findings suggest that allopurinol preconditioning renal IRI (Figure 1).
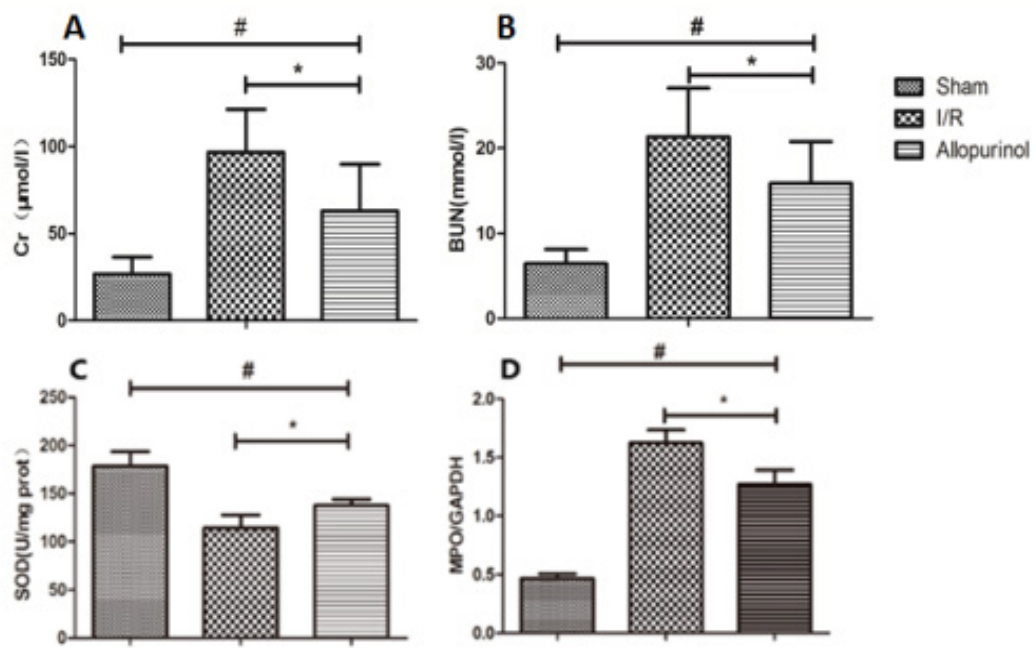

FIGURE 1 - Allopurinol preconditioning can significantly improve renal function in I/R injury. (A, B) Compared with the I/R group, rats preconditioned with allopurinol showed significantly reduced BUN and serum creatine levels. (C, D) Compared with the I/R group, allopurinol preconditioning significantly inhibited MPO and increased SOD levels; ${ }^{*} \mathrm{p}<0.05$ vs. the I/R group; \# $\mathrm{p}<0.05$ vs. the sham group.

\section{Allopurinol preconditioning improved morphologi- cal feature after renal IRI}

Based on our analysis of H\&E stained sections, renal $\mathrm{I} / \mathrm{R}$ resulted in significant renal injury, including tubular cell apoptosis and medullary hemorrhage. By contrast, allopurinol preconditioning significantly ameliorated this severe renal damage (Figure 2). Using the Jablonski scoring scale, $30 \mathrm{~min}$ renal ischemia followed by $72 \mathrm{~h}$ reperfusion resulted in severe acute tubular necrosis, whereas allopurinol preconditioning could significantly reduce tissue pathology $(\mathrm{p}<0.05)$.

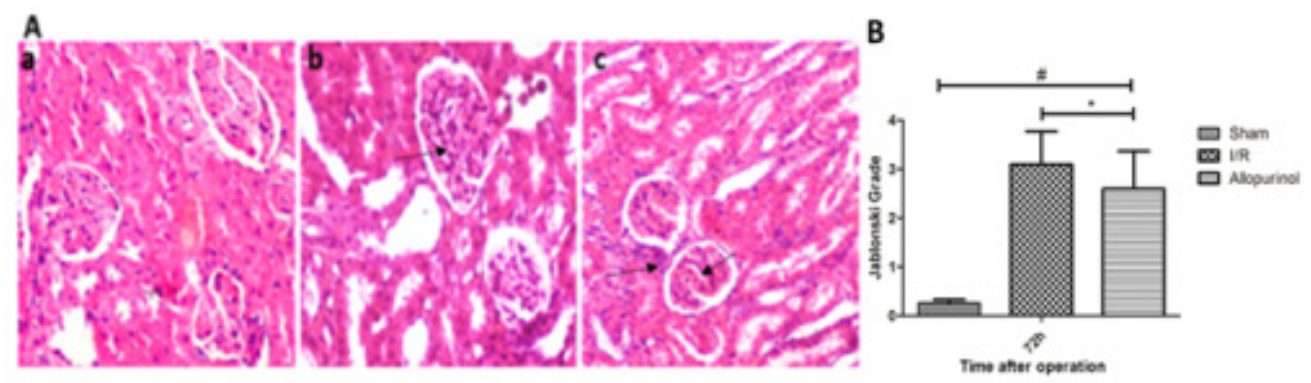

FIGURE 2 - Allopurinol preconditioning improved morphological features after renal IRI. (A) H\&E stained sections. Allopurinol preconditioning significantly ameliorated the severe I/R-induced renal damage, including tubular necrosis, medullary hemorrhage, and congestion (a-Sham group, $b-\mathrm{I} / \mathrm{R}$ group, c-Allopurinol group). (B) Based on an analysis of Jablonski scores, allopurinol preconditioning resulted in the downregulation of Jablonski scores; ${ }^{*} \mathrm{p}<0.05$ vs. the $\mathrm{I} / \mathrm{R}$ group; \# $\mathrm{p}<0.05$ vs. the sham group.

\section{Allopurinol preconditioning inhibited apoptosis af- ter renal IRI}

Using the TUNEL assay, we found that allopurinol preconditioning treatment reduced I/R-induced apoptosis.
The apoptosis index in allopurinol preconditioning groups was significantly lower than that in the $I / R$ group (Figure 3 ). Additionally, the levels of caspase- 3 and Bax were significantly increased in the I/R group compared with the sham group, whereas Bcl-2 expression was down-regulated in $\mathrm{I} / \mathrm{R}$ group 
$(\mathrm{p}<0.05)$. Notably, allopurinol preconditioning treatment reduced

expression of Bcl-2 (Figure 4; $\mathrm{p}<0.05$ ).

the changes in the levels of Bax and caspase- 3 , and restored the
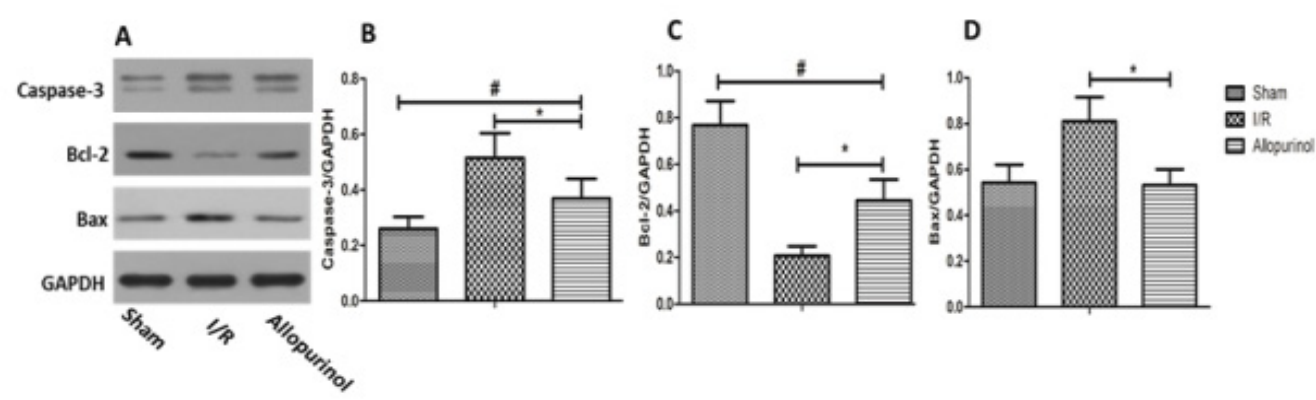

FIGURE 3 - Allopurinol preconditioning inhibited apoptosis following renal IRI. (A-C) TUNEL assays revealed that allopurinol preconditioning reduced renal tubule epithelial cell apoptosis (a-Sham group, b-I/R group, c-Allopurinol group). (D) The apoptosis index in the allopurinol preconditioned group was significantly lower than that in the I/R group; * $\mathrm{p}<0.05$ s. the I/R group.

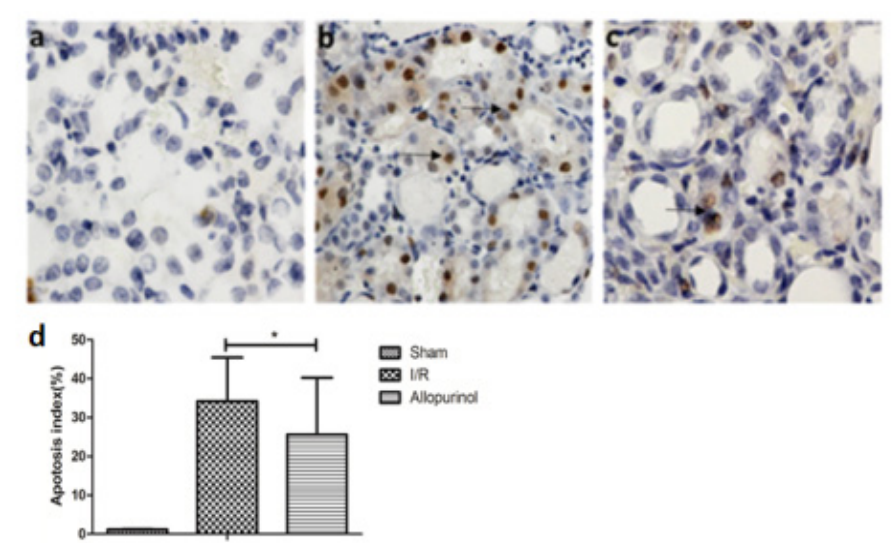

FIGURE 4 - Allopurinol preconditioning inhibited the expression of pro-apoptosis associated molecules and promoted the up-regulation of apoptosis inhibitory proteins in I/R model. (A) Compared with the sham group, levels of caspase-3 and Bax were significantly increased in the $\mathrm{I} / \mathrm{R}$ group, and $\mathrm{Bcl}-2$ expression was down-regulated in the I/R group. However, allopurinol preconditioning resulted in reduced levels of Bax and caspase-3, and restored the expression levels of Bcl-2. (B-D) Compared with GAPDH expression levels, Bax, Bcl-2, and Caspase-3 expression were determined after allopurinol preconditioning treatment, as shown in A. The amount of inhibition of Caspase-3 and Bax expression were $43.9 \%$ and $41.8 \%$, while Bcl-2 expression was increased by $112.6 \%$; $* \mathrm{p}<0.05$ vs. the $\mathrm{I} / \mathrm{R}$ group; $\# \mathrm{p}<0.05$ vs. the sham group.

\section{Allopurinol preconditioning reduces inflammatory marker expression after renal IRI}

HMGB1 is a novel marker of inflammation in IRI. Using western blotting and immunohistochemistry, we observed that HMGB1 expression was inhibited by allopurinol preconditioning administered prior to renal ischemic reperfusion (Figure 5, $\mathrm{p}<0.05)$.
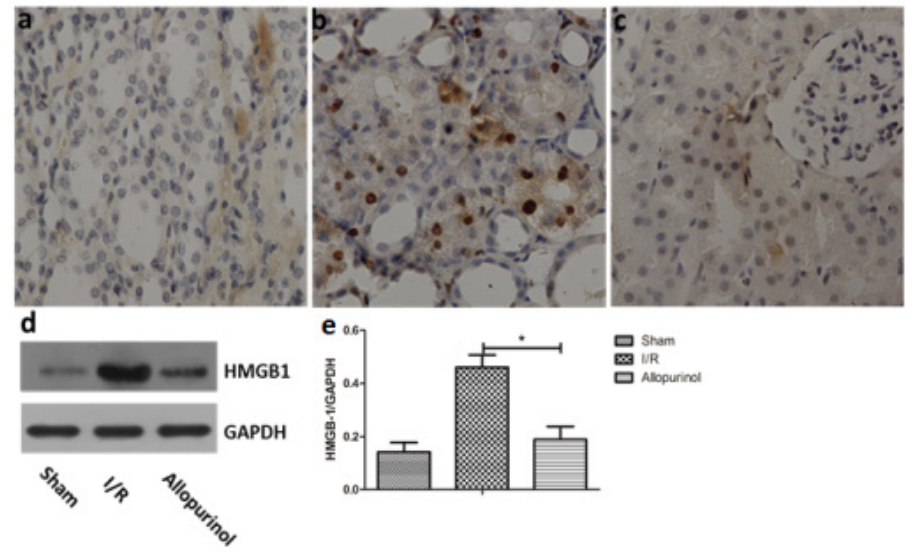

FIGURE 5 - Allopurinol preconditioning down-regulated the expression of an inflammatory marker after renal IRI. (A-C) Immunohistochemical detection of HMGB1 expression revealed that it was inhibited by allopurinol preconditioning prior to renal ischemia reperfusion (a-Sham group, b-I/R group, c-Allopurinol group). (D) Western blotting revealed that HMGB1 expression was inhibited by allopurinol preconditioning. (e) Compared with GAPDH expression levels, HMGB-1 expression was determined after allopurinol preconditioning, as shown in (D). The expression levels of HMGB1 were inhibited by $54.6 \%$; ${ }^{*} \mathrm{p}<0.05 v s$. the I/R group.

\section{Discussion}

A kidney allograft from a donor who died from brain or cardiac death may undergo a longer ischemic time because of unstable hemodynamic circulation ${ }^{18}$. Acute kidney injury prior to donation can also increase primary graft dysfunction and result in delayed graft function. Significant efforts to evaluate the optimal treatment of acute kidney injury prior to donation have been ongoing for many years. Currently, it is thought that 
preconditioning therapy based on drug and physical methods may offer optimal ways to relieve IRI ${ }^{19}$.

The production of ROS and intracellular $\mathrm{Ca}^{2+}$ overload represent two major pathophysiological components of IRI ${ }^{20}$. Some studies have shown that xanthine oxidase (XO) plays an important role in ROS production and intracellular $\mathrm{Ca}^{2+}$ overload in an I/R model $^{21}$. Allopurinol has been the main medicine used to treat gout since 1963. Recently, it also has shown radical scavenging and cardioprotective effects in a myriad of cardiovascular conditions. Allopurinol has been used in studies of heart ischemia and reperfusion because of its reported activity to improve IRI of vascular endothelial cells. Allopurinol, an isomer of hypoxanthine, is an inhibitor of XO. Therefore, it may inhibit $\mathrm{I} / \mathrm{R}$ injury by this mechanism ${ }^{21}$. In our present study, allopurinol preconditioning also restored renal function caused by $30 \mathrm{~min}$ IRI (Figure 1).

Allopurinol exhibits antianginal activity in a rat model. In a vasopressin-induced angina model, oral administration of allopurinol exhibited effects that were remarkably analogous to those of amlodipine. Effects on the levels of endothelial NO synthase (eNOS) expression and serum nitrate were the main underlying mechanism ${ }^{22}$. All of these mechanisms can exert similar effects in kidney IRI. Thus, in our present study, levels of MDA and SOD were significantly improved in rats pretreated with allopurinol compared with the I/R group.

Apoptosis is another mechanism that can lead to organ damage throughout the course of renal ischemia/reperfusion. This observation suggests that a significant component of inflammation could be induced by apoptosis after renal ischemia/ reperfusion injury. Based on morphological analyses, allopurinol preconditioning could improve tubular necrosis, medullary hemorrhage and congestion based on H\&E staining (Figure 2).

Many studies have suggested that members of the Bcl2 family, especially Bcl-2 and Bax, are key physiological and pathological regulators of apoptosis that act via the activation of caspase-triggered signaling cascades ${ }^{23}$. Some studies have shown that oxidative stress could stimulate nuclear factor-kappa $\mathrm{B}(\mathrm{NF}-\kappa \mathrm{B})$ activation, which has been associated with the rapid transcription of TNF- $\alpha$ and induction of apoptosis. Postcon is known to attenuate myocardial apoptosis and reduce Caspase- 3 activity, which may be mediated by the inhibition of the oxidantactivated NF- $\kappa \mathrm{B}-\mathrm{TNF}-\alpha$ signaling pathway ${ }^{24}$. In our present study, allopurinol preconditioning inhibited pro-apoptosis-associated molecule expression and induced the up-regulation of the inhibitor of apoptosis protein in our I/R model (Figures 3 and 4). I/Rinduced tissue injury has been associated with the activation of the MAPK signaling pathways ${ }^{25}$.
The release of high-mobility group box-1 (HMGB1) and other DAMP molecules from dying cells may promote organ dysfunction and inflammation in renal $\mathrm{IRI}^{26}$. According to recent reports, UA can induce the extracellular release of HMGB1 and subsequently enhance the production of HMGB1, which can lead to aggravation of the inflammatory response by promoting the translocation of HMGB1 from nucleus to the cytoplasm ${ }^{14}$. Thus, suppression of UA production during IRI may inhibit the subsequent inflammatory response generated by UA and the subsequent response triggered by HMGB1 nuclear translocation. In this present study, we also established that the allopurinol preconditioning could inhibited the expression of HMGB-1 (Figure 5).

\section{Conclusions}

Allopurinol exhibits protective effects against renal injury induced by renal ischemia/reperfusion. The main underlying mechanism might be related to the inhibition of apoptosis and the nuclear translocation of the inflammatory marker HMGB1. Our findings may provide some evidence for clinical preconditioning with allopurinol to protect renal function. Finally, a HMGB1 inhibition may represent a potential therapeutic strategy for the treatment of ischemia-reperfusion injury.

\section{References}

1. Vallon V, Osswald H. Adenosine receptors and the kidney. Handb Exp Pharmacol. 2009;(193):443-70. PMID: 19639291.

2. Prieto-Moure B, Caraben-Redano A, Aliena-Valero A, Cejalvo D, Toledo AH, Flores-Bellver M, Martinez-Gil N, Toledo-Pereyra LH, Lloris Carsi JM. Allopurinol in renal ischemia. J Invest Surg. 2014;27(5):304-16. PMID: 24914485.

3. Khader A, Yang WL, Kuncewitch M, Prince JM, Marambaud P, Nicastro J, Coppa GF, Wang P. Novel resveratrol analogues attenuate renal ischemic injury in rats. J Surg Res. 2015;193(2):80715. PMID: 25214260

4. Jeong EK, Jang HJ, Kim SS, Oh MY, Lee DH, Eom DW, Kang KS, Kwan HC, Ham JY, Park CS, Jang DS, Han DJ. Protective effect of eupatilin against renal ischemia-reperfusion injury in mice. Transplant Proc. 2015;47(3):757-62. PMID: 25891726.

5. Tsung A, Zheng N, Jeyabalan G, Izuishi K, Klune JR, Geller DA, Lotze MT, Lu L, Billiar TR. Increasing numbers of hepatic dendritic cells promote HMGB1-mediated ischemia-reperfusion injury. J Leukoc Biol. 2007;81(1):119-28. PMID: 17062605.

6. Rabadi MM, Ghaly T, Goligorksy MS, Ratliff BB. HMGB1 in renal ischemic injury. Am J Physiol Renal Physiol. 2012;303(6):F873-85. PMID: 22759395.

7. Zhu P, Xie L, Ding HS, Gong Q, Yang J, Yang L. High mobility group box 1 and kidney diseases (Review). Int $\mathrm{J}$ Mol Med. 2013;31(4):763-8. PMID: 23440289.

8. Musumeci D, Roviello GN, Montesarchio D. An overview on HMGB1 inhibitors as potential therapeutic agents in HMGB1- 
related pathologies. Pharmacol Ther. 2014;141(3):347-57. PMID: 24220159 .

9. Rabadi MM, Kuo MC, Ghaly T, Rabadi SM, Weber M, Goligorsky MS, Ratliff BB. Interaction between uric acid and HMGB1 translocation and release from endothelial cells. Am J Physiol Renal Physiol. 2012;302(6):F730-41. PMID: 22189943.

10. George J, Carr E, Davies J, Belch JJ, Struthers A. High-dose allopurinol improves endothelial function by profoundly reducing vascular oxidative stress and not by lowering uric acid. Circulation. 2006;114(23):2508-16. PMID: 17130343.

11. Lee WY, Koh EJ, Lee SM.Acombination of ischemic preconditioning and allopurinol protects against ischemic injury through a nitric oxide-dependent mechanism. Nitric Oxide. 2012;26(1):1-8. PMID: 22119149.

12. Kang SM, Lim S, Song H, Chang W, Lee S, Bae SM, Chung JH, Lee H, Kim HG, Yoon DH, Kim TW, Jang Y, Sung JM, Chung NS, Hwang KC. Allopurinol modulates reactive oxygen species generation and $\mathrm{Ca}^{2+}$ overload in ischemia-reperfused heart and hypoxia-reoxygenated cardiomyocytes. Eur J Pharmacol. 2006;535(1-3):212-9. PMID: 16516885.

13. Wen SH, Li Y, Li C, Xia ZQ, Liu WF, Zhang XY, Lei WL, Huang WQ, Liu KX. Ischemic postconditioning during reperfusion attenuates intestinal injury and mucosal cell apoptosis by inhibiting JAK/STAT signaling activation. Shock. 2012;38(4):411-9. PMID: 22777122.

14. Salvadori M, Rosso G, Bertoni E. Update on ischemia-reperfusion injury in kidney transplantation:Pathogenesis and treatment. World J Transplant. 2015;5(2):52-67. PMID: 26131407.

15. Lee WY, Lee SM. Synergistic protective effect of ischemic preconditioning and allopurinol on ischemia/reperfusion injury in rat liver. Biochem Biophys Res Commun. 2006;349(3):1087-93. PMID: 16959212.

16. Chen H, Xing B, Liu X, Zhan B, Zhou J, Zhu H, Chen Z. Similarities between ozone oxidative preconditioning and ischemic preconditioning in renal ischemia/reperfusion injury. Arch Med Res. 2008;39(2):169-78. PMID: 18164960.

17. Jablonski P, Howden BO, Rae DA, Birrell CS, Marshall VC, Tange J. An experimental model for assessment of renal recovery from warm ischemia. Transplantation. 1983;35(3):198-204. PMID: 6340272.

18. Allen MB, Billig E, Reese PP, Shults J, Hasz R, West S, Abt PL. Donor hemodynamics as a predictor of outcomes after kidney transplantation from donors after cardiac death. Am J Transplant 2016 Jan;16(1):181-93. doi: 10.1111/ajt.13432.19. McCafferty K, Forbes S, Thiemermann C, Yaqoob MM. The challenge of translating ischemic conditioning from animal models to humans: the role of comorbidities. Dis Model Mech. 2014;7(12):1321-33. PMID: 25481012.

20. Abdallah Y, Kasseckert SA, Iraqi W, Said M, Shahzad T, Erdogan A, Neuhof C, Gündüz D, Schlüter KD, Tillmanns H, Piper HM, Reusch HP, Ladilov Y. Interplay between $\mathrm{Ca} 2+$ cycling and mitochondrial permeability transition pores promotes reperfusion-induced injury of cardiac myocytes. J Cell Mol Med. 2011;15(11):2478-85. PMID: 21199327.

21. Bussmann AR, Marton Filho MA, Modolo MP, Modolo RP, Amado P, Domingues MA, Castiglia YM, Modolo NS. Effect of allopurinol on the kidney function, histology and injury biomarker (NGAL, IL 18) levels in uninephrectomised rats subjected to ischaemiareperfusion injury. Acta Cir Bras. 2014;29(8):515-21. PMID: 25140593.

22. Al-Zahrani YA, Al-Harthi SE, Khan LM, El-Bassossy HM, Edris SM, A Sattar MA.The possible antianginal effect of allopurinol in vasopressin-induced ischemic model in rats. Saudi Pharm J. 2015;23(5):487-98. PMID: 26594114.
23. Shin JH, Chun KS, Na YG, Song KH, Kim SI, Lim JS, Kim GH. Allopurinol protects against ischemia/reperfusion-induced injury in rat urinary bladders. Oxid Med Cell Longev. 2015;2015:906787. PMID: 26491537.

24. Kin H, Wang NP, Mykytenko J, Reeves J, Deneve J, Jiang R, Zatta AJ, Guyton RA, Vinten-Johansen J, Zhao ZQ. Inhibition of myocardial apoptosis by postconditioning is associated with attenuation of oxidative stress-mediated nuclear factor-kappa B translocation and ha release. Shock. 2008;29(6):761-8. PMID: 18496137.

25. Yin T1, Sandhu G, Wolfgang CD, Burrier A, Webb RL, Rigel DF, Hai T, Whelan J. Tissue-specific pattern of stress kinase activation in ischemic/reperfused heart and kidney. J Biol Chem. 1997;272(32):19943-50. PMID: 9242662.

26. Lau A, Wang S, Liu W, Haig A, Zhang ZX, Jevnikar AM. Glycyrrhizic acid ameliorates HMGB1-mediated cell death and inflammation after renal ischemia reperfusion injury. Am J Nephrol. 2014;40(1):84-95. PMID: 25059568.

\section{Correspondence:}

Tao Qiu

Department of Organ Transplantation, Renmin Hospital of Wuhan University

ZiYang Road 99, Wuhan, Hubei, China, 430060

Phone: +86 $02788041911-82235$

Fax: +86 02788042292

mdqiutao@yahoo.com

Received: Nov 16, 2015

Review: Jan 14, 2016

Accepted: Feb 15, 2016

Conflict of interest: none

Financial sources: National Natural Science Foundation of China (No. 81400753), Hubei Natural Science Foundation (No. 2014CFB362), the bureau of public health of Hubei Province (JX6B14), and the project of Wuhan Municipal Science and Technology Bureau (2013062301010808).

${ }^{1}$ Research performed at Key Laboratory, Hubei Province for Digestive System Disease, Department of Gastroenterology, Renmin Hospital, Wuhan University, Hubei, China. 


\section{Erratum}

Manuscript: Allopurinol preconditioning attenuates renal ischemia/reperfusion injury by inhibiting HMGB1 expression in a rat model

Publication: Acta Cir Bras. 2016;31(3):176-82.

DOI: http://dx.doi.org/10.1590/S0102-865020160030000005

On page 176 of the original publication, where it is written ${ }^{\mathrm{I}} \mathrm{PhD}$, Department of Organ Transplantation, Renmin Hospital of Wuhan University, Wuhan, Hubei, China. Conception and design of the study, acquisition and analysis of data, manuscript writing.

IIPhD, Department of Organ Transplantation, Renmin Hospital of Wuhan University, Wuhan, Hubei, China. Design and supervised all phases of the study, critical revision.

IIIPhD, Physician, Department of Urology, Renmin Hospital of Wuhan University, Wuhan, Hubei, China. Acquisition of data.

${ }^{\text {IV }}$ Physician, Intensive Care Unit, PuAi Hospital, Anlu, Hubei, China. Helped processing figures.

It should read

${ }^{\mathrm{I}} \mathrm{PhD}$, Department of Organ Transplantation, Renmin Hospital of Wuhan University, Wuhan, Hubei, China. Conception and design of the study, acquisition and analysis of data, manuscript writing.

IIPhD, Department of Organ Transplantation, Renmin Hospital of Wuhan University, Wuhan, Hubei, China. Design and supervised all phases of the study, critical revision.

IIIPhD, Physician, Department of Urology, Renmin Hospital of Wuhan University, Wuhan, Hubei, China. Acquisition of data.

${ }^{\text {IV }}$ Physician, Intensive Care Unit, PuAi Hospital, Anlu, Hubei, China. Helped processing figures.

Jiang-qiao Zhou and Tao Qiu contributed equally to this work as co-first authors. 